\title{
Rêves brûlants
}

\section{W. Bauer}

Dr méd., président de l'Institut suisse pour la formation médicale postgraduée et continue (ISFM)

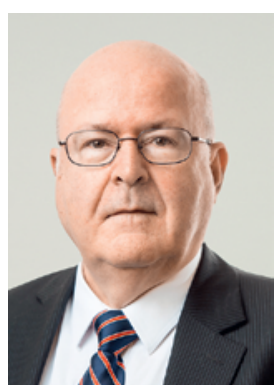

Un coup d'œil à mon agenda m’a rappelé sans ambiguïté que la rédaction attend un «Et encore...». C'est normalement un plaisir de pouvoir exposer ses idées et impressions aux lecteurs du Bulletin des médecins. Mais là, il fait chaud, tout simplement trop chaud! Les rues miroitent, rares sont les nuages qui obscurcissent le soleil et aucun coup de tonnerre n'ose se transformer en véritable orage. Sans compter qu'il règne une atmosphère de vacances propice au rêve. Moi en tout cas, j'ai succombé au rêve, peut-être parce que j'ai dû écrire ces lignes au bord de la mer Égée, où la chaleur, la lumière et les ondes reflétées sont encore plus intenses que sur le lac de Zurich.

C'est ainsi que je rêvais d'un système de santé auquel le corps médical, conjointement avec toutes les autres professions, apporterait une contribution engagée et soucieuse de la qualité. Mais aussi d'un système de santé dans lequel les conditions-cadres seraient conçues de manière à ce que le personnel puisse s'acquitter de ses tâches avec satisfaction, en se sachant considéré et en jouissant de perspectives positives.

Je rêvais de ressources suffisantes pour la formation initiale et postgraduée. Le service et l'activité scientifique sont importants, mais une valeur au moins égale devrait être accordée à l'enseignement. Participer à la formation médicale ne serait pas vu comme un devoir mais comme un privilège et bénéficierait d'un soutien concret des directions et autorités. Il ne s'agit pas d'un fardeau, mais de l'avenir de la qualité en médecine.

Je rêvais que toutes les parties concernées tombaient d'accord pour trouver une réglementation de la limitation du temps de travail plus raisonnable que celle qui existe aujourd'hui et qui est en partie correctement et en partie mal mise en œuvre. Il ne serait en aucun cas question de revenir sur les acquis d'une limitation du temps de travail qui améliore la qualité de vie, mais les médecins à tous les niveaux hiérarchiques disent souvent lors des visites qu'un assouplissement intelligent pourrait faciliter la vie de tous.

Je rêvais que les nouveaux systèmes informatiques ne seraient pas seulement mis en place parce que nous vivons à l'ère de ces systèmes et que les responsables de l'hôpital de Seldwyla, par exemple, auraient l'impression d'être archaïques s'ils n'introduisaient pas un «système d'information hospitalier Seldwyla 001» dès que possible. Les systèmes informatiques sont sans aucun doute indispensables, mais pour l'instant, leur introduction entraîne encore, dans trop de bureaux de médecins-assistants à Seldwyla et ailleurs, des énervements et un surcroît de travail, parce que la convivialité n'était pas l'un des critères essentiels du projet.

Je rêvais que l'expression "evidence based» ne deviendrait pas vide de sens, mais qu'en tant qu'«evidence based medicine» et qu'«evidence based politics», elle serait traitée avec tout le sérieux qu'elle mérite. Mais si l'on fait preuve de réalisme, les données dont disposent les médecins sont souvent insuffisantes pour prendre une décision véritablement fondée sur des faits. La prise de décision conjointe avec des patients bien informés est donc d'autant plus importante. Ce "Clinical reasoning» ainsi que la communication correspondante devraient être enseignés et développés de manière plus ciblée.

Il est malheureusement plus que douteux que le rêve de décisions politiques fondées sur des faits se réalise un jour. L'activisme, le besoin de se positionner, la réticence au compromis et la rigidité idéologique sont des pierres d'achoppement sur la voie de décisions réfléchies et au service d'une cause. Un exemple d'actualité? L'intervention dans les tarifs, censée favoriser les soins médicaux de base mais qui entraîne en fait des pertes financières insupportables dans les hôpitaux pédiatriques, comme c'était prévisible!

Je rêvais aussi que notre société ne considérait pas avant tout les personnes âgées comme des problèmes et des charges financières et ne développait pas leur prise en charge en s'appuyant essentiellement sur des manuels de gestion d'entreprise. Bien sûr que les homes, les organismes des soins à domicile et autres institutions doivent être efficacement structurés et solidement financés. Mais il serait bon de nous rappeler qu'il s'agit de la génération qui a construit notre société actuelle et qui mérite que dans chaque institution, il y ait du temps pour une conversation empathique au-delà des quelques minutes allouées par le manuel du bureau de conseil en gestion.

Il me restait bien d'autres choses à rêver quand la réalité a soudain repris ses droits avec cette question: pourquoi tant de rêves portent-ils sur des thèmes qui sont en fait des évidences? Quelqu'un peut-il me l'expliquer? 